\title{
Tras las pistas en los procesos de pensamiento, un acercamiento desde la acción pedagógica
}

Following the clues in the thought processes, an approach from the pedagogical action

Lucía Garcia ${ }^{1}$

Garcia, Lucia I miradas $\mathrm{N}^{\circ} 14$ - 2016. ISSN: 0122 994X Págs 61 - 70

Recepción: Junio 18 de 2016 Aprobación: Octubre 01 de 2016

Publicación: Diciembre 20 de 2016

\section{Resumen:}

El presente artículo hace parte de la propuesta de investigación adelantada en los estudios de Doctorado en Educación y Pensamiento Educativo - Universidad Tecnológica de Pereira, la cual pretende contextualizar desde la acción pedagógica a los procesos de pensamiento a través de la búsqueda en las bases de datos y con ello reflexionar sobre el papel de la educación en la injerencia de los mismos, así como profundizar en los trabajos adelantados para establecer conexiones posibles entre procesos de pensamiento y conciencia sintética, como una aproximación a la teoría de Peirce.

Palabras Clave: Procesos de pensamiento, metacognición, comunicación, mediaciones habilidades de pensamiento.

1 Licenciada en Biología y Química por la Universidad de Caldas. Magister en Comunicación Educativa por la Universidad Tecnológica de Pereira. Docente tiempo completo Básica primaria, municipio de Pereira. milugarcia@utp.edu.co lucytag@yahoo.com 


\section{Abstract:}

This article is part of the research proposal advanced in the studies of Doctorate in Education and Educational Thought at the Technological University of Pereira, which seeks to contextualize from the pedagogical action to the thought processes through the search in the bases of Data and thus reflect on the role of education in their interference, as well as to deepen the work advanced to establish possible connections between thought processes and synthetic consciousness, as an approximation to Peirce's theory.

Key Words: Processes of thought, metacognition, communication, mediations, thinking skills.

\section{Procesos de pensamiento}

\section{Antecedentes del problema}

En el presente documento se hace un análisis por los diferentes trabajos de investigación que han abordado el tema procesos de pensamiento, permitiendo desde sus respectivas miradas un acercamiento a sus enfoques, metodologías y conclusiones parciales, lo que origina otras maneras de entender el asunto en discusión.

\section{Procesos de pensamiento como objeto de análisis y discusión.}

Se consultaron las bases de datos suscritas a la Universidad Tecnológica y Google Académico, encontrándose, tesis doctorales, artículos de revistas indexadas y artículos de revistas especializadas, las palabras claves con las cuales se realizó la búsqueda para el presente documento fueron:

- Educación y procesos de pensamiento

- Comunicación y procesos de pensamiento
- Mediaciones y procesos de pensamiento

Obteniéndose la siguiente estadística de documentos relacionados con el tema $\mathrm{y}$ donde $\mathrm{R}$ es la cantidad de documentos hallados en cada base de datos.

Gráfico $\mathrm{N}^{\mathrm{o}} 1$ Resultados hallados según la base de datos consultada

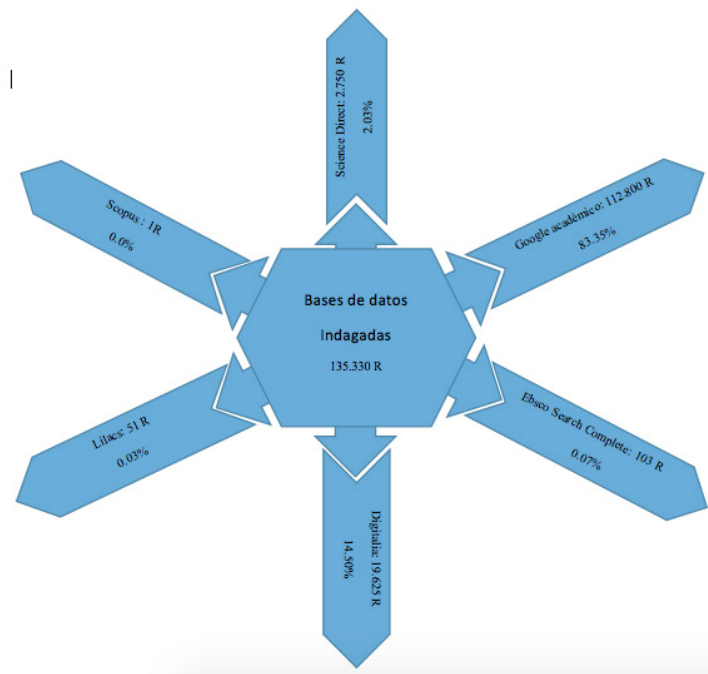

Fuente: Elaboración propia

Posterior a esta búsqueda se seleccionaron 67 documentos los cuales corresponden al objeto de análisis y discusión, selección que obedece a los siguientes criterios:

- Relación con el tema procesos de pensamiento

- Artículos que evidencian un acercamiento a los procesos de pensamiento a través de la educación, comunicación y las mediaciones.

El abordaje de los respectivos documentos se realizó bajo la orientación de la siguiente pregunta: ¿Qué concepciones, marcos teóricos y enfoques se han desarrollado para investigar los procesos de pensamiento? 
Tabla N ${ }^{\circ} 1$ Criterios de búsqueda

\begin{tabular}{|c|c|c|c|}
\hline Criterio de búsqueda & $\begin{array}{l}\mathrm{N}^{\mathrm{a}} \text { de } \\
\text { artículos }\end{array}$ & $\begin{array}{ll}\mathrm{N}^{o} & \text { de } \\
\text { tesis } & \end{array}$ & Capítulo \\
\hline $\begin{array}{lr}\text { Educación } & y \\
\text { procesos } & \text { de } \\
\text { pensamiento } & \end{array}$ & 25 & 1 & 1 \\
\hline $\begin{array}{l}\text { C o m u n i c a c i ó n } \\
\text { y procesos de } \\
\text { pensamiento }\end{array}$ & 11 & 0 & \\
\hline $\begin{array}{l}\text { M e d i a c i o n e s } \\
\text { y procesos de } \\
\text { pensamiento }\end{array}$ & 29 & 0 & \\
\hline
\end{tabular}

\section{Educación y procesos de pensamiento}

Se seleccionaron 25 artículos, 1 tesis doctoral y 1 capítulo de libro. Dichos documentos se procesaron a través del uso del software NVIVO, el cual permite la visualización de categorías de palabras que son referenciadas en todos los documentos como se muestra a continuación en el gráfico.

Gráfico $N^{\circ} 2$ Palabras asociadas a la categoría educación y procesos de pensamiento

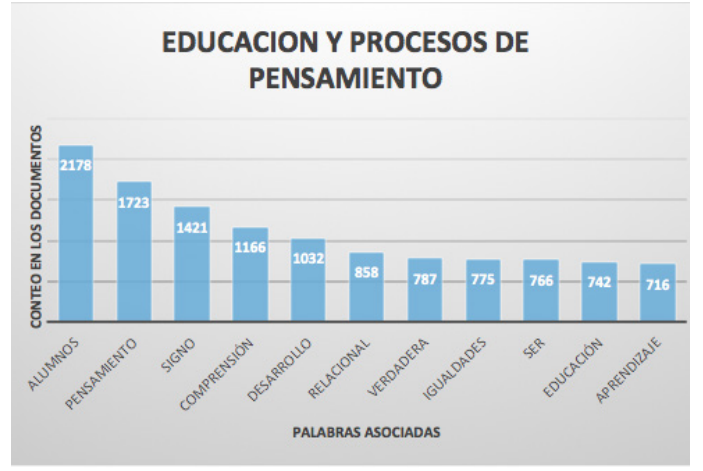

Los valores numéricos corresponden a la cantidad de veces que dicha palabra es referida en los 27 documentos seleccionados.

La educación y procesos de pensamiento en los documentos en cuestión, se relaciona con el hecho que para el desarrollo del pensamiento, la educación como espacio es un lugar privilegiado para tal fin y en sintonía con ello en el artículo " Enseñanza de procesos de pensamiento: Metodología, Metacognición y transferencias", se evidencia la necesidad de pasar de la enseñanza del conocimiento declarativo, a una enseñanza del conocimiento procesual y procedimental, considerada esta última de gran importancia para el desarrollo integral del estudiante, afirmándose allí que:

"Es absolutamente preciso
hacer consciente al alumno
de los procesos que se
emplean en la elaboración de
conocimientos, facilitándole
por todos los medios la
reflexión metacognitiva
sobre las habilidades de
conocimiento, los procesos
cognitivos, el control y la
planificación de la propia
actuación y de los otros,
la toma de decisiones y la
comprobación de resultados"
(M.E.C., 1989b, p.99). 2

Lo que genera pensamiento crítico y habilidades metacognitivas, ya que el estudiante es consciente de su propio proceso de aprendizaje, que tras la tutela del docente y a través de su cambio de actitud logran que este sea participe y autónomo en la toma de decisiones, potenciando de esta manera los ambientes favorables en la educación como espacio primordial para el afianzamiento de los procesos de pensamiento.

En nuestro país el Ministerio de Educación Nacional en el documento lineamientos curriculares para el área de matemáticas, distingue cinco tipos de pensamientos que

2 Martín I, Juan Francisco. Enseñanza de procesos de pensamiento: Metodología y transferencias. Dpto. de Didáctica, Organización y Métodos de Investigación. Universidad de Salamanca 
deben se desarrollados en los estudiantes a fin de lograr potenciar los procesos de pensamiento y ellos son:

- Pensamiento numérico y sistemas numéricos: cuyo énfasis está en el uso de la aritmética, el poder pensar en los números y usarlos en contextos significativos.

- Pensamiento espacial y sistemas geométricos: como forma de exploración y representación del espacio.

- Pensamiento métrico y sistemas de medida: como continuidad para cuantificar numéricamente las magnitudes que surgen en las construcciones de representaciones geométricas y espaciales.

- Pensamiento aleatorio y los sistemas de datos: referido a la resolución de problemas a través del tratamiento de la estadística y la probabilidad.

- Pensamiento variacional y sistemas algebraicos y analíticos: como integración de todos los pensamientos para analizar, organizar y modelar matemáticamente situaciones problémicas que impliquen a las ciencias así como a la actividad practica del hombre.

Por su parte en el documento de tesis doctoral ${ }^{3}$ se hace referencia al pensamiento relacional como (Gonzáles, 2006)

$\begin{array}{lr}\text { La actividad } & \begin{array}{r}\text { intelectual } \\ \text { (interna) }\end{array} \\ \text { consistente } \\ \text { en examinar objetos y } \\ \text { situaciones } & \text { matemáticas, } \\ \text { considerándolos remo } & \text { como }\end{array}$

3 Gonzáles M, Marta. Desarrollo de pensamiento relacional y comprensión del signo igual por alumnos de tercero de educación primaria. Granada 2006

\begin{abstract}
totalidades, detectar de manera espontánea o buscar relaciones entre ellos $y$ utilizar dichas relaciones con una intencionalidad, es decir, para alcanzar un objetivo.
\end{abstract}

Este pensamiento en el contexto de las matemáticas y específicamente en la aritmética, permite a los estudiantes adquirir habilidades para establecer relaciones de igualdad, comparación y transformación, indispensables para la comprensión de la estructura del sistema de numeración decimal y las operaciones aritméticas; todo ello como patrones para las generalizaciones posteriores.

El desarrollo del pensamiento ligado al pensar y tomado este como proceso dirigido que permite resolver problemas, es decir, pensar es lo que pasa en la mente de un sujeto cuando resuelve un problema, que en relación al párrafo anterior se evidencia en la medida que los estudiantes resuelven satisfactoriamente las igualdades y sentencias aritméticas, genera mayores desempeños tras la adquisición de habilidades cognoscitivas.

Por su parte al hablar de procesos de pensamiento se encuentra que son los componentes activos de la mente y por lo tanto son elementos básicos para construir, organizar y usar los conocimientos y en este orden de ideas los procesos mentales superiores -planificación, supervisión, evaluacióny retroalimentación, contribuyen a las habilidades de pensamiento como fruto de los procesos de pensamiento.

En el documento: Pensamiento Crítico: ¿Qué es y por qué es importante? ${ }^{4}$, el autor expresa el consenso frente a la definición expuesta para el pensamiento crítico, otro tipo de pensamiento diferente a los

4 FACIONE, Peter. Pensamiento Crítico: ¿Qué es y por qué es importante? Insight Assessment 2007. http://www. insightassessment.com 
expuestos en párrafos anteriores, el cual es una habilidad cognitiva en donde lo esencial es la interpretación, análisis, inferencia, explicación y autorregulación.

"Entendemos que el
pensamiento crítico (PC)
es el juicio auto regulado y
con propósito que da como
resultado interpretación,
análisis, evaluación e
inferencia, como también
la explicación de las
consideraciones de evidencia, conceptuales, metodológicas, criteriológicas o contextuales en las cuales se basa ese juicio. El PC es fundamental como instrumento de investigación. Como tal, constituye una fuerza liberadora en la educación $y$ un recurso poderoso en la vida personal y cívica de cada uno. Si bien no es sinónimo de buen pensamiento, el PC es un fenómeno humano penetrante, que permite auto rectificar. "El pensador crítico ideal es una persona que es habitualmente inquisitiva; bien informada; que confía en la razón; de mente abierta; flexible; justa cuando se trata de evaluar; honesta cuando confronta sus sesgos personales; prudente al emitir juicios; dispuesta a reconsiderar y si es necesario a retractarse; clara con respecto a los problemas o las situaciones que requieren la emisión de un juicio; ordenada cuando se enfrenta a situaciones complejas; diligente en la búsqueda de información relevante; razonable en la selección de criterios; enfocado en

preguntar, indagar, investigar; persistente en la búsqueda de resultados tan precisos como las circunstancias y el problema o la situación lo permitan. Así pues, educar buenos pensadores críticos significa trabajar en pos de este ideal. Es una combinación entre desarrollar habilidades de pensamiento crítico y nutrir aquellas disposiciones que consistentemente producen introspecciones útiles y que son la base de una sociedad racional y democrática" (pág.21)

Hasta el momento en los documentos analizados se encuentra que los diferentes tipos de pensamientos, como habilidades cognitivas son susceptibles a ser enseñados, y de esta manera potenciar los procesos mentales, por consiguiente la educación es fundamental en cuanto permite centrar estrategias que desarrollen actividades encaminadas a la enseñanza de los mismos procesos de pensamiento.

\section{Comunicación $y$ procesos de pensamiento}

Se seleccionaron 11 artículos, que al ser procesados arrojan las palabras relevantes en el conjunto de documentos.

Gráfico $N^{\circ} 3$ Palabras asociadas a la categoría comunicación y procesos de pensamiento

COMUNICACION Y PROCESOS DE PENSAMIENTO

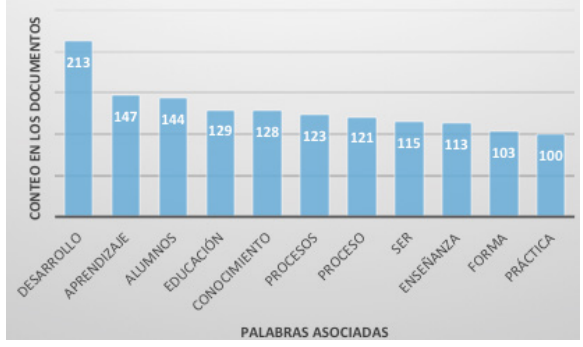

Revista de Investigación 
Ésta categoría en los documentos analizados se encuentran en relación con los procesos cognoscitivos a través de la acción didáctica desarrollada en el aula, es así como se establece una interacción comunicativa que potencia el desarrollo del pensamiento, el cual contribuirá a los requerimientos de la sociedad actual, la sociedad del conocimiento.

En uno de los documentos referidos al desarrollo del pensamiento lógico matemático, se evidencia que éste como proceso puede ser enseñado y que tal acción se inicia desde la comprensión del concepto, paso indispensable para establecer las posibles relaciones con los procedimientos, los cuales deben dotarse de significado, el que será transferido en el sentido de uso de cada operación.

En este sentido en el documento estrategias de enseñanza - otra mirada al quehacer en el aula ${ }^{5}$, se explora sobre la importancia de las estrategias de enseñanza que el docente propone en clase, ya que pueden favorecer algún tipo particular de comunicación e intercambio tanto intrapersonal como entre los alumnos y el profesor, y entre cada alumno y el grupo. Definiendo el término de estrategias como el conjunto de decisiones que asume el docente para orientar la enseñanza y así promover el desarrollo cognoscitivo de sus estudiantes.

\section{Mediación y procesos de pensamiento}

Se seleccionaron 29 documentos los cuales se observan a través del software ya mencionado y con las palabras relevantes halladas en los mismos se procede al análisis.

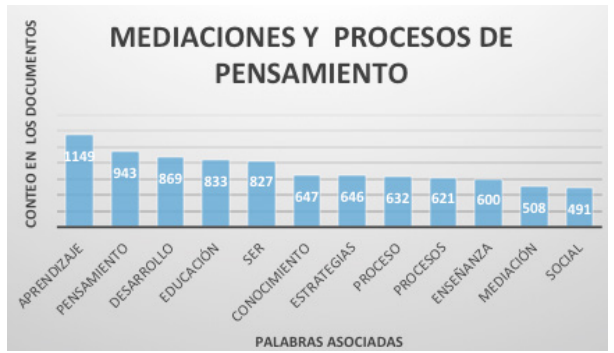

Gráfico No 4 Palabras asociadas a la categoría mediaciones y procesos de pensamiento

En el análisis de las palabras asociadas se evidencia que los documentos seleccionados se relacionan con la mediación como un desencadenante de lo cognitivo, es decir, una buena mediación permite el desarrollo cognitivo en los estudiantes, es así como el juego de roles potencia el desarrollo del pensamiento al igual que la integración de las TIC a los procesos de enseñanza.

En este sentido la Metacognición como palabra asociada hace referencia a la cognición sobre la cognición, al conocimiento propio del conocimiento, pero referida especialmente a la toma de conciencia, a la autorregulación y en relación al enfoque constructivista los estudiantes construyen sus propios conocimientos, situaciones y significados, y al mismo tiempo evalúan y reconocen sus propios procesos de reconstrucción y este orden de ideas la complementariedad entre metacognición y constructivismo pueden conducir al desarrollo del pensamiento.

En donde el pensar posibilita esas nuevas maneras de ver el mundo, de reconocer las diferentes cosmovisiones, en interacción con la computadora como mediador emergente en la sociedad del conocimiento. Y donde cada vez es más apremiante el desarrollo del pensamiento desde edades tempranas, lo que facilita la autonomía en la toma de decisiones. 
Y en palabras de Schmeck, aprender es aprender a pensar y de esta manera todo aprendizaje será significativo en la medida que sea mediado socialmente y requiere de unos procesos para que éste se dé, los cuales dependen de dos situaciones, la primera es que si bien en el aprendizaje existen procesos, estos a su vez se pueden realizar de diversas maneras y la segunda hace alusión al hecho de que pueden ser iniciados desde la propia situación de enseñanza aprendizaje bien por el estudiante o por el profesor.

Es así entonces que se plantean los siguientes procesos como congruentes entre las diferentes posturas y ampliamente desarrollados por Beltrán:

- Sensibilización: se asume como inicial para cualquier aprendizaje y se compone de la motivación, la emoción y las actitudes

- Atención: es ella quien filtra la información que será almacenada, tanto en calidad como en cantidad.

- Adquisición: la comprensión, pesentándose la comprension, - retención - y transformación. Una vez se incorpora el material informativo que es significativo para el sujeto, éste le da sentido al comprenderlo y entra a hacer parte de él de manera permanente, lo que no quiere decir q ellos sean estáticos, por el contrario son objeto de transformación.

- Personalización y control: a través de éste el sujeto asume la responsabilidad de su aprendizaje.

- Recuperación: hacer uso del material que previamente fue categorizado, organizado para su almacenaje.

- Transfer: es capacidad de aplicar lo aprendido en diferentes contextos
- Evaluación: permite verificar el logro de los objetivos y a su vez fortalece al sujeto aumentando la motivación y el auto concepto.

\section{Habilidades de pensamiento}

Son la base de la interacción comunicativa y conforman los procesos de pensamiento más generales, las habilidades pueden ser innatas o adquiridas, ellas corresponden a un conjunto de operaciones de carácter principalmente cognitivo. Es en este sentido donde la mediación juega un papel vital a través de la activación de los procesos del aprendizaje, los que en últimas contribuirán a la mejora en los procesos de pensamiento, toda vez que se promueva la metacomprensión como reconocimiento de los propios procesos cognitivos y de los métodos empleados para esos mismos procesos.

En relación a la teoría de Peirce, se plantea que todo nuestro mundo es susceptible a ser comprendido y por supuesto el desarrollo de habilidades de pensamiento aporta elementos para tal fin, refiriéndose entonces al pensamiento como elemento esencial del signo, el cual opera desde la lógica tríadica y se evidencia en el mundo a través de la acción, fruto de los procesos de pensamiento como proceso sígnico,

\section{Metodología}

La metodología propuesta en el presente documento corresponde al análisis de contenido, los archivos seleccionados para abordar el estado en cuestión son sistematizados a través del software NVIVO, del cual se extrae la información relevante para posteriores análisis, es así como se visualizan las referencias de cada palabra asociada en los diferentes documentos. 
Tabla N² Palabras asociadas "mediación"

\begin{tabular}{|l|l|}
\hline Nombre del documento consultado & Referencias \\
\hline $\begin{array}{l}\text { Ábaco abierto desarrollo procesos de } \\
\text { pensamiento }\end{array}$ & 1 \\
\hline $\begin{array}{l}\text { De los modelos pedagógicos a } \\
\text { procesos de pensamiento }\end{array}$ & 18 \\
\hline $\begin{array}{l}\text { Dialnet-DesarrolloDeHabilidadesMetac } \\
\text { ognitivasConElUsoDeLas-3006962 }\end{array}$ & 1 \\
\hline $\begin{array}{l}\text { Dialnet-LaComputadoraComoMediado } \\
\text { rSimbolicoDeAprendizajesEs-1280128 }\end{array}$ & 8 \\
\hline $\begin{array}{l}\text { dinámica del aprendizaje y la } \\
\text { mediación }\end{array}$ & 2 \\
\hline $\begin{array}{l}\text { Desarrollo de competencias cognitivas } \\
\text { en el aula }\end{array}$ & 3 \\
\hline $\begin{array}{l}\text { ensenar-a-pensar-de-forma-personal- } \\
\text { primeros-pasos }\end{array}$ & 1 \\
\hline formación ciudadana & 4 \\
\hline $\begin{array}{l}\text { Georgina Vivanco aprendizaje } \\
\text { transmediado }\end{array}$ & 2 \\
\hline Guía para desarrollo de pensamiento & 49 \\
\hline $\begin{array}{l}\text { Hacia un nuevo paradigma del } \\
\text { aprendizaje estratégico }\end{array}$ & 1 \\
\hline $\begin{array}{l}\text { hacia una pedagogía para la creatividad } \\
\text { y el desarrollo del pensar }\end{array}$ & 5 \\
\hline $\begin{array}{l}\text { juego de rol como mediaciones en el } \\
\text { desarrollo del lenguaje y pensamiento } \\
\text { matemático }\end{array}$ & 9 \\
\hline
\end{tabular}

Posterior a este análisis de visualizan las Categoría de palabras, las cuales permiten identificar en qué contexto se hace referencia a la palabra seleccionada y con este insumo se construyen las fichas para el análisis de contenido.

Tabla $N^{o} 3$ Categoría de palabras "mediación"

Referencia 38 - Cobertura $0,01 \%$
computadora. Ellas son: mediación cognosci-
tiva, mediación cultural, mediaciones de refer-
encia, mediaciones
Referencia 39 - Cobertura $0,01 \%$
mediaciones institucionales, mediaciones vide-
otecnológicas.

- La mediación valorativa - cognitiva está referida a

Referencia 40 - Cobertura 0,01\%

proceso de manera particular.

- La mediación cultural toma cuerpo desde el

Referencia 41 - Cobertura 0,01\%

contempladas o enmarcadas en la mediación cultural. De todas maneras posiblemente

\section{Referencia 42 - Cobertura $0,01 \%$}

importancia de los procesos de mediación en la construcción de la

Tabla No 4 Ficha análisis de contenido

\begin{tabular}{|l|l|l|l|}
\hline Código: & $\begin{array}{l}\text { Fuente: } \\
\text { Artículos de } \\
\text { investigación }\end{array}$ & Fecha: & Categoría: \\
EA-PP-0055 $22 / 2016$ & metodologías \\
\hline
\end{tabular}

Pregunta referencial: ¿Qué concepciones, marcos teóricos y enfoques se han desarrollado para investigar los procesos de pensamiento?

Realizador: Lucia García

Unidad de análisis: frases o palabras que indican o se refieren a la categoría sobre la que se está indagando

Mediación

Unidad de contexto: donde se encuentra la unidad de análisis en la frase o palabra a la que se hace mención.

andamios para el aprendizaje, la mediación pedagógica

DEL PENSAMIENTO se concibe como mediación pedagógica

juegos de rol son la mediación educativa para desarrollar el pensamiento

La mediación es un proceso comunicacional

que en el proceso de mediación, el docente enfatiza la internalización 
1. Relación de unidad de análisis y la unidad de contexto

andamios para el aprendizaje, la mediación pedagógica

DEL PENSAMIENTO se concibe como mediación pedagógica

juegos de rol son la mediación educativa para

desarrollar el pensamiento

La mediación es un proceso comunicacional

que en el proceso de mediación, el docente enfatiza la internalización

Observaciones: en los documentos analizados existe una relación entre el pensamiento y la mediación pedagógica como acto comunicativo.

Las fichas de análisis de contenido son reagrupadas, según información que arroje la primera lectura y a partir de allí se inicia el cruce de datos con las teorías abordadas en la presente investigación.

\section{Conclusiones}

- Los procesos de pensamiento son retomados en los documentos analizados como una forma de potenciar las habilidades matemáticas los cuales pueden ser aprendidos $\mathrm{y}$ enseñados a través de la implementación de diversas metodologías.

- $\mathrm{Si}$ bien es cierto que los procesos de pensamiento se pueden enseñar, en el caso colombiano porqué estos no se evidencian en las pruebas internacionales.

- Los procesos de pensamiento se evidencian desde la terceridad, como una posibilidad de mediación.

- En los documentos analizados se establece una relación entre la educación y la comunicación con los procesos cognoscitivos, relacionados estos con los conocimientos declarativos, mientras que la mediación esta en concordancia con los procesos cognitivos en relación a los conocimientos procedimentales.

\section{Referencia Biliográfica}

Beltrán, J. (1998). Procesos, Estrategias y Técnicas de Aprendizaje. Madrid: Síntesis, S.A.

Restrepo J. Mary luz. SER - SIGNO - INTERPRETANTE. Filosofía de la Representación de Charles S. Peirce. Ediciones: Significantes de Papel. Santa Fe de Bogotá, 1993.

Restrepo J. Mary luz. Representación, relación tríadica en el pensamiento de Charles S. Peirce. Universidad Nacional de Colombia.

Martín I, Juan Francisco. Enseñanza de procesos de pensamiento: Metodología y transferencias. Dpto. de Didáctica, Organización y Métodos de Investigación. Universidad de Salamanca. Recuperado de: http://www.uv.es/RELIEVE/v7n2/ RELIEVEv7n2 2.pdf

Gonzáles M, Marta. Desarrollo de pensamiento relacional y comprensión del signo igual por alumnos de tercero de educación primaria. Granada 2006. Recuperado de: https://dialnet.unirioja.es/servlet/tesis?codigo $=1210$ 
Lucía García

Facione, Peter. Pensamiento Crítico: ¿Qué es y por qué es importante? Insight Assessment 2007. Recuperado de: http://www. insightassessment.com

Rivas, U. (11 de agosto de 1999).

Recuperado en: http://www.unav.es/gep/

Signo.html. 